\title{
Aprendiendo a investigar en la Educación Superior: elaboración de preguntas científicas y su tratamiento metodológico ${ }^{1}$
}

\section{Learning to do research in Higher Education: formulation of scientific questions and its methodological resolution}

\author{
Carmen Viejo² \& Rosario Ortega-Ruiz ${ }^{3}$
}

Fecha de recepción: 20/06/2019; Fecha de revisión: 27/07/2019; Fecha de aceptación: 30/09/2019

Cómo citar este artículo:

Viejo, C. \& Ortega-Ruiz, R. (2019). Aprendiendo a investigar en la Educación Superior: elaboración de preguntas científicas. Revista de Innovación y Buenas Prácticas Docentes, 8(3), 1-10.

\section{Autor de Correspondencia: cviejo@uco.es}

\begin{abstract}
Resumen:
Uno de los retos más importantes en el contexto de la Educación Superior actual es el fomento de competencias en el alumnado. Concretamente, en los estudios de postgrado, se hace necesario formar a los y las estudiantes para que sean capaces de enfrentar tareas de carácter científico, prestando especial atención a las competencias de alto nivel cognitivo, presentes en tareas de revisión de textos científicos y análisis crítico de argumentaciones, diseño de la investigación, análisis estadístico de datos, inferencia de conclusiones y elaboración de informes de investigación, así como el manejo informático. Este proyecto ha tenido la finalidad de implementar una metodología activa que promueva la transferencia del conocimiento adquirido por el alumnado en cada una de las materias específicas del título, a la aplicación práctica y desarrollo competencial necesario para la elaboración del Trabajo Final de Máster (TFM). Los resultados del proyecto mostraron un aumento progresivo de las competencias del alumnado egresado en el máster a medida que el curso avanzaba, si bien no todas las competencias siguieron un mismo patrón en su evolución; mientras que algunas tuvieron un rápido desarrollo durante los primeros meses de formación, sufriendo luego cierto estancamiento, otras evolucionaron de forma más lenta. Estos resultados apuntan a la importancia de la puesta en marcha de planes de trabajo centrados en el desarrollo competencial de los alumnos y alumnas.
\end{abstract}

Palabras clave: desarrollo competencial, enseñanza postgrado, intervención metodológica, trabajo fin de máster.

\begin{abstract}
:
One of the most important challenges in the context of current Higher Education is the promotion of competencies in university students. Specifically, in postgraduate levels, it is necessary to train students to be able to face scientific tasks, paying special attention to high-level cognitive skills, present in tasks of review of scientific texts and critical analysis of argumentations, research design, statistical data analysis, inference of conclusions and preparation of research reports, as well as computer management. The project had the purpose of implementing an active methodology that promotes the transfer of the knowledge
\end{abstract}

\footnotetext{
${ }^{1}$ Este artículo se deriva del proyecto de Innovación Docente titulado "La Investigación en el marco de la Educación Superior: desarrollo de competencias para la elaboración de preguntas científicas y su tratamiento metodológico" (2017-1-4014), obtenido en la convocatoria2017/18 del Plan de Innovación y Buenas Prácticas Docentes (modalidad 1). En este proyecto participaron, además de las autoras de este artículo, el siguiente profesorado de la Universidad de Córdoba (por orden alfabético): Juan Calmaestra, José Antonio Casas, Francisco Córdoba, Daniel Falla, Olga Gómez, Mercedes Gómez-López, Rocío Luque, Antonio J. Rodríguez-Hidalgo y Eva Romera.

2 Universidad de Córdoba (España).cviejo@uco.es

3 Universidad de Córdoba (España). ed1orrur@uco.es
} 
acquired by the students in each of the specific subjects of the degree, to the practical application and competence development necessary for the preparation of the Master's thesis (TFM). The results showed a progressive increase of the students' competences as the course progressed, although not all the competences followed the same pattern in their evolution; while some had a quick development during the first months of training, suffering then some standstill, others evolved more slowly. These results point to the importance of the implementation of teaching plans focused on the students' competence development.

Keywords: Competential development, Higher Education, Master Thesis, Methodological reform. 


\section{INTRODUCCIÓN}

La Universidad es una de las instituciones sociales más antiguas y que más prestigio social tiene, y ello es debido, en gran medida, al reconocimiento de que junto a la tarea de transmisión del conocimiento, tiene la de creación del propio conocimiento. El marco de convergencia europea que supuso la escueta legislación común europea que se concretó en el llamado proyecto Bolonia, activó muchos de los procesos implícitos en la vida universitaria. Uno de ellos, no muy reconocido todavía, se refiere a la articulación de las dos grandes tareas universitarias: la creación de conocimiento mediante la investigación científica, y la transmisión de competencias para que generación tras generación esto sea posible. Es decir, la formación para llegar a ser un/a científico/a. En los últimos tiempos, la universidades europeas han tenido que afrontar un nuevo reto: dibujar un marco común de trabajo que diera respuesta a la necesidades reales de los y las ciudadanas del siglo XXI; este reto suponía establecer de forma muy clara lo que sería el marco de referencia, el Espacio Europeo de Educación Superior.

El Informe Bricall (2000) destacaba la Universidad como institución social a la que se le atribuían un sinfín de funciones; no obstante, estas funciones podían resumirse en tres: la función socializadora, dedicada a la preservación y transmisión crítica del conocimiento, cultura y valores sociales; la función orientadora, dedicada al desarrollo de las capacidades de cada individuo; y la función investigadora y de extensión cultural, con la que se pretende el aumento de la base del conocimiento de la sociedad. La Declaración de Lisboa (2007), ya señalaba que "el cambio climático, los problemas energéticos, el aumento de la longevidad, la rápida evolución de la tecnología, la interdependencia socio-económica a escala mundial y las crecientes desigualdades económicas dentro de Europa y entre Europa y otros continentes: todos estos temas necesitan de la investigación básica y aplicada para transformar el conocimiento en innovación tecnológica y social. Así será posible resolver los problemas a medida que surjan, asegurando simultáneamente prosperidad económica y estabilidad social en los distintos países". En este sentido, la educación universitaria ha de asumir un rol protagonista en cuanto se refiere a la formación de profesionales competentes en un mundo cambiante, capaces de transformar el conocimiento especializado adquirido en la Universidad en herramientas para elaborar respuestas a las necesidades sociales. La European Universities Association (2017) subraya este aspecto, dejando constancia explícita del papel principal que juega la Universidad en cuanto a la investigación e innovación, siendo este un eje en el desarrollo de políticas, y actividades y proyectos.

Este marco general de indicaciones cristaliza en los diferentes títulos y programas concretos de las materias que se ofertan en cada Universidad. Por tanto, el reto de las Universidades Europeas finalmente supone asumir una serie de cambios que abogan por la transformación de los procesos de enseñanza-aprendizaje que deben ir más allá de metodologías tradicionales encaminadas a la mera transmisión de conocimientos; ello supone el diseño de un plan formativo que, en el caso de los másteres, se concreta en lograr que las y los egresados sean plenamente competentes para construir su propio conocimiento profesional y estar en condiciones de asumir la formación para construir conocimiento científico (objetivo de los estudios de doctorado). Se trata de equipar al alumnado de postgrado de una serie de herramientas estratégicas necesarias para que tomen conciencia de sus capacidades y también de sus necesidades, y se enfrenten al proceso de aprendizaje de la tarea investigadora de forma más competente y progresivamente más autónoma. Educar en competencias implica conseguir unos aprendizajes globales útiles para poder resolver con eficacia los 
problemas que se plantean a nivel social (Pérez-Gómez, 2010; Moral-López, ArrabalGómez y González-López, 2010).

El Proyecto de Innovación Docente que nos planteamos y del que esta memoria expresa resultados, trata de contribuir a resolver esta tarea de forma satisfactoria; se suma así al reto propuesto del trabajo de las competencias para el fomento del conocimiento y la construcción de sólidas propuestas de investigación que contribuyan al avance en los problemas sociales de nuestro siglo. El Trabajo Final de Máster (TFM), como parte de la formación de postgrado, es de una relevancia extraordinaria en cuanto que significa, o puede significar si se realiza adecuadamente, el ejercicio del tránsito del manejo del conocimiento científico como un producto externo, a la construcción de un conocimiento propio que permite al estudiante disponer de su propia autonomía como elaborador de conocimiento propio. Si bien, para llegar a resolver esta tarea de forma satisfactoria, la formación a nivel de máster debe centrarse no solo en el desarrollo de contenidos propios de cada materia, sino en el conjunto de competencias necesarias para abordar un trabajo de esta índole. En este sentido, los docentes que participamos en este proyecto, asumimos la labor de modelado de las estrategias necesarias para que el alumnado sea capaz de asumir el control de su propio proceso de aprendizaje y, concretamente, de su tarea de elaboración de un TFM que suponga el fruto de los aprendizajes desarrollados durante los estudios de máster.

\section{DESARROLLO DE LA EXPERIENCIA DE INNOVACIÓN}

Este Proyecto de Innovación Docente se propone como finalidad última el implementar, de forma coordinada entre diferentes docentes delMáster de Psicología Aplicada a la Educación y el Bienestar -Dpto. de Psicología, Universidad de Córdoba-, una metodología activa que favorezca la transferencia del conocimiento teórico especializado adquirido en las materias del título, a la aplicación práctica y desarrollo competencial necesario para la elaboración del Trabajo Fin de Máster. Esto se concreta en los siguientes objetivos:

1. Implementar, de manera coordinada, metodologías activas dirigidas al avance del desarrollo competencial del alumnado en tareas profesionales e investigadoras propias del nivel de Máster, comparando el grado de desarrollo competencial antes y después de la intervención y valorando los resultados obtenidos.

2. Gestionar, de forma progresiva y explícita, la transferencia del conocimiento teórico especializado a la aplicación práctica en la experiencia de realización del TFM.

\subsection{Material y Métodos}

Siguiendo con la dinámica de trabajo que en cierto modo se había comenzado a desarrollar de forma espontánea, la implementación de este proyecto supondrá la constitución en una estructura en red de los diferentes profesores y profesoras implicados en algunas de las asignaturas del Máster Investigador de Psicología Aplicada a la Educación; esto permitirá que se articulen distintas experiencias en cada una de las materias, previamente coordinadas, dirigidas a estimular en el alumnado el deseo de realizar un TFM que los entrene en las tareas de investigación y creación del conocimiento.

\subsection{Participantes}

Es preciso tener en cuenta que el Máster anteriormente citado, el cual se beneficia directamente del desarrollo de este proyecto, se estructura en dos cursos académicos, concentrando la actividad académica en tres cuatrimestres. Por ello, las actividades que se diseñan se estructuran a lo largo de todo el proceso de formación, si bien, poniendo el mayor peso de intervención en los inicios, cuando el alumnado tiene una mayor carencia y necesidad de un desarrollo competencial que hasta ahora no había requerido. 
De esta forma, los participantes en este proyecto y, por tanto, beneficiarios directos de acción llevada a cabo, han sido los egresados y los titulados del Máster de Psicología Aplicada a la Educación y el Bienestar, de la Universidad de Córdoba, durante el curso 2017/18. Este alumnado representa la tercera promoción de egresados al máster ( $\mathrm{N}=34)$; aunque la intervención se lleva a cabo a lo largo de los tres cuatrimestres de duración del máster y, por ende, solo en el segundo curso podría considerarse que la intervención habría finalizado, la mayoría de las acciones se contemplan durante este primer curso por lo que se presentan resultados preliminares correspondientes a este primer curso(está previsto volver a tomar datos en el siguiente curso, cuando la intervención esté finalizando).

\subsection{Intervención metodológica}

En los másteres de orientación hacia la investigación, como es el Máster de Psicología al que se dirige este proyecto, el TFM consiste en la elaboración y ejecución de un pequeño proyecto de investigación que, con independencia de su mayor o menor complejidad, está destinado a entrenar las competencias investigadoras de los egresados. Para contribuir a que el alumnado aborde esta tarea en las mejores condiciones competenciales posibles, un grupo de profesores y profesoras docentes de este título hemos iniciado una reforma metodológica que apuesta por establecer un modelo de orientación, supervisión y tutorización de los TFM de los estudiantes de los que nos responsabilizamos, dándole especial relevancia a la puesta en práctica de las competencias cognitivas y procedimentales que, previamente, hemos abordado de forma explícita, a través de diversos talleres competenciales desde nuestra materias teóricas. siguiente:

El plan de actividades ligadas al proyecto de Innovación Docente ha sido el

1. Talleres propios de cada asignatura (ver Viejo y Ortega-Ruiz, 2018 para la revisión del diseño de los talleres puestos en marcha): cada una de las asignaturas implicadas ha llevado a cabo un taller específico de desarrollo competencial. Para ello, se ha coordinado la acción de los profesores y profesoras implicados que, en sesiones de 2 horas aproximadamente, implementaron dentro de sus propias asignaturas y en relación al contenido de estas, un taller específico diseñado para el desarrollo progresivo de las competencias identificadas como necesarias para el TFM. Estos talleres se llevan a cabo durante el primer y segundo cuatrimestre de máster.

2. Talleres específicos: se han diseñado dos talleres específicos dirigidos a dos habilidades transversales a cualquier proceso de investigación: el trabajo metacognitivo (Aprender de forma estratégica: la importacia de la metacognición para el desarrollo competencial) y la habilidades de formulación y presentación de la investigación (Defensa de un TFM: competencias para la exposición pública de la investigación). Estos talleres se llevan a cabo en sesiones largas de trabajo, en el segundo y tercer cuatrimestre de máster, respectivamente, y en sesiones individuales con el alumnado que presente su TFM para orientaciones particulares si lo requieren (en el tercer cuatrimestre).

3. Evaluación continua y final del desarrollo competencial y metacognitivo: se llevan a cabo hasta 5 momentos de recogida de datos a lo largo del proceso de formación del alumnado, coincidentes con el principio y final de cada cuatrimestre (actualmente, solo 3 de ellas se han llevado a cabo). De esta forma, se valora el desarrollo progresivo de estas competencias. El objetivo último, aun por desarrollar, es la valoración también del resultado final expresado en la valoración cualitativa que el 
tribunal experto, externo al máster, efectúa de los TFMs (se realizará cuando los estudiantes foco de la innovación defiendan su TFM).

Se han llevado a cabo regularmente sesiones para el trabajo de coordinación (presenciales y telemáticas) de acuerdo a las actividades previstas en este proyecto, y de acuerdo a la temporalización de cada una de las materias (materias de primer año y de segundo año), apostando por un desarrollo progresivo de las competencias requeridas.

\subsection{Evaluación de la intervención}

Si bien aún no se han presentado y defendido los TFMs del alumnado beneficiario de esta acción y, por tanto, no es posible llevar a cabo una evaluación global y precisa de la intervención desarrollada -contando con la valoración cuantitativa y cualitativa que realiza el tribunal evaluador de TFMs del Máster (siempre constituido por profesores no docentes, sino académicos externos provenientes de otras universidades) expertos en las temáticas del máster, se han realizado diferentes medidas de consecución de competencias con objeto de conocer la posible evolución de las mismas. Para ello, se ha utilizado una adaptación de un instrumento post-hoc para la evaluación de competencias investigadoras, que cuenta con una serie de 35 ítems, medidos con una escala continua de diez puntos (1-10), que valora el grado de percepción sobre la adquisición de distintas competencias cognitivas y procedimentales -revisión de textos científicos y análisis crítico de argumentaciones, diseño de la investigación, análisis de datos, inferencia de conclusiones y elaboración de informes de investigación, y manejo informático- (Viejo y Ortega, 2018).

\section{RESULTADOS}

El diseño longitudinal de la evaluación de esta intervención nos permite reportar los resultados preliminares derivados de los 3 primeros momentos de toma de datos (principio de curso, final del 1er cuatrimestre, final del 2do cuatrimestre). Los resultados que se presentan son, por tanto, parciales y deben valorarse con cautela, sin embargo, manifiestan logros que son reseñables.

Los ítems del cuestionario fueron agrupados en torno a 5 factores que constituyen las grandes categorías competenciales contempladas: (1) revisión de textos científicos y análisis crítico de argumentaciones, (2) diseño de la investigación, (3) análisis de datos, (4) inferencia de conclusiones y elaboración de informes de investigación), y (5) manejo informático. La figura 1 muestra los resultados obtenidos.

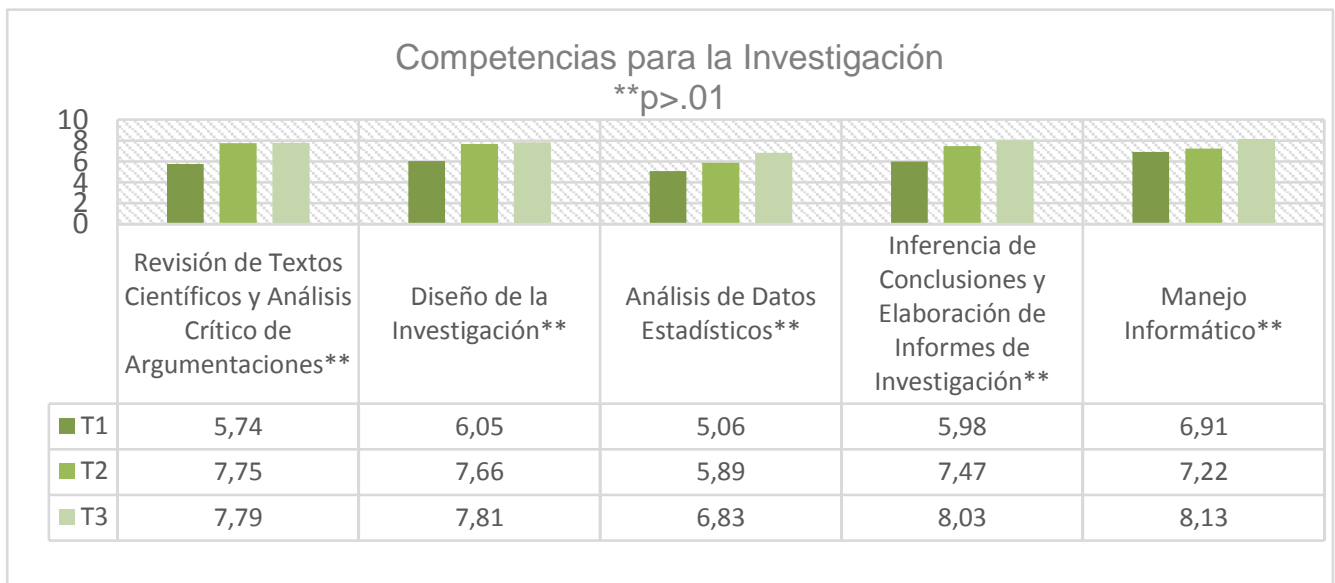

Figura 1. Puntuaciones medias en las competencias para la investigación en los 3 primeros tiempos de medida. 
Los resultados mostraron que ha existido un aumento progresivo de la competencia del alumnado egresado en el máster a medida que el curso avanzaba, manifestado en la puntuación de los tres T del longitudinal. El nivel de partida de todas las competencias oscilaba entre puntuaciones de 5.06 (competencia para el análisis de datos) y 6.91 (competencia para el manejo informático). En el T3, sin embargo, el nivel competencial para la investigación oscilaba entre el 6.83 de la competencia para el análisis de datos, la cual mantiene las puntuaciones más bajas en todos los tiempos; y el 8.13 de la competencia para el manejo informático, la cual también partía de los puntajes más altos.

Para verificar la significatividad estadística de estas diferencias, se llevó a cabo un análisis ANOVA de medidas repetidas, tomando como factor los distintos tiempos de medida realizados. En todos los casos resultó existir diferencias significativas entre algunos de los tiempos de medida.

En cuanto a la escala de Revisión de textos científicos y análisis crítico de argumentaciones, el test de Mauchly's señaló la violación de la hipótesis de esfericidad $(\chi 2(2)=21.765, p=.000)$ por lo que los grados de libertad fueron corregidos con el estadístico de esfericidad de Huynh-Feldt $(\varepsilon=.71)$. Los resultados señalaron diferencias estadísticamente significativas $(F(1.43,110.37)=65.79, p=.000)$; el estadístico de Bonferroni indicó que estas se producían del T1 a los T2 y T3 de las medidas

En cuanto a la escala de Diseño de la investigación, el test de Mauchly's señaló la aceptación de la hipótesis de esfericidad $\left(\chi^{2}(2)=2.50, p=.29\right)$. Los resultados de esfericidad asumida señalaron diferencias estadísticamente significativas ( $F(2,86.195)$ $=52.382, \mathrm{p}=.000)$; el estadístico de Bonferroni indicó que estas diferencias se establecían, como en el caso anterior, entre el primer tiempo de medida y los T2 y T3.

En la tercera de las competencias analizadas, la referida al Análisis de datos, se asume de nuevo la esfericidad de los datos $\left(\chi^{2}(2)=.170, p=.918\right)$, siendo que los resultados de esfericidad asumida indicaron diferencias $(F(2,62.27)=31.14, p=.000)$; el estadístico de Bonferroni indicó que, en este caso, las diferencias se establecían entre el T3 y las dos anteriores, siempre en gradiente creciente del T1 al T2 y T3.

En cuanto a la escala de Inferencia de Conclusiones y elaboración de informes de investigación, se asumió la esfericidad de los datos según indicaba el test de Mauchly's $(\chi 2(2)=.987, p=.610)$ y se obtuvieron resultados que señalaron diferencias estadísticamente significativas $(F(2,100.19)=52.63, p=.000)$; en esta ocasión el estadístico de Bonferroni indicó que estas diferencias se producían entre todos los tiempos de medida.

Por último, en relación a la escala de Manejo informático, el test de Mauchly's señaló la aceptación de la hipótesis de esfericidad $(\chi 2(2)=.415, p=.81)$. Los resultados de esfericidad asumida señalaron diferencias estadísticamente significativas ( $F(2$, $36.05)=17.62, p=.000$ ); el estadístico de Bonferroni indicó que estas diferencias se establecían desde el T3 y los dos T anteriores.

\section{CONCLUSIONES}

El planteamiento y desarrollo de este proyecto descansaba sobre las bases teóricas y empíricas de trabajos diseñados y desarrollados previamente que dibujaban el perfil de necesidades que el alumnado de Máster tiene respecto a su formación investigadora; 
una formación que, recogiendo los enunciados de la declaración de Bergen y de la declaración de London, respectivamente, marcan el imperativo de que los estudios de postgrado se distingan de los de grado básicamente en relación a la competencia general de aumentar la capacidad para diseñar el propio proceso cognitivo en términos de aumentar el dominio sobre la toma de decisiones autónoma sobre cómo organizar el conocimiento propio. Los resultados previos que hemos ido observando derivados de un cambio en cuanto al enfoque del proceso de enseñanza-aprendizaje en estos nivel de Educación Superior apuntaban la bondad de un modelo metodológico basado en la búsqueda de la activación expresa de competencias cognitivas, procedimentales y actitudinales de carácter transversal y de nivel superior, en términos de conocimiento científico, para el tránsito de una formación universitaria básica a un nivel de formación intermedia de postgrado, y de ahí, si se desea, a una verdadera autonomía investigadora. Solo así se permitirá actualizar las competencias necesarias para el inicio de los estudios de doctorado al tiempo que se activan procesos útiles para asumir la actividad profesional de forma independiente, con criterios propios, con pensamiento abierto y capacidad crítica basada en la correcta lectura de la evidencia científica. Solo de esta forma adquiere verdadera relevancia el periodo intermedio de formación de postgrado que denominamos máster. Para lo que aquí interesa, este elemento debe estar en el interior de las decisiones docentes de un master investigador como es el que nos ocupa.

Los resultados concretos derivados de esta iniciativa investigadora ligada a la innovación docente deben tomarse aun con cautela, ya que los dos últimos tiempos de medida aun no están registrados, pero el análisis que se ha realizado sobre el progreso de las competencias seleccionadas a lo largo de las medidas de un longitudinal, de momento de tres tiempos, muestran indicadores de progreso que conviene señalar. Las limitaciones que supone la no implementación aun completa del programa de formación, así como el reducido número de alumnado que participa de un Máster como este, no permiten generalizar y extraer conclusiones determinantes. Sin embargo, si podrían entenderse como un buen punto de partida para realizar una reflexión estimulante sobre la metodología docente desplegada, muy especialmente la que se refiere a la innovación del trabajo articulado de varias asignaturas actuando conjuntamente en orden a objetivos comunes. Sin evitar la autocrítica, no cabe duda de que la linea de innovación resulta productiva y exitosa al menos en términos del progreso que los estudiantes realizan en las cinco competencias que previamente se habían detectado (Viejo y Ortega-Ruiz, 2018) como relevantes en relación a la formación inicial para la investigación, que por otro lado son las que se supone que se desarrollan con la ejecución del TFM. En este sentido, es preciso destacar algunos de los principales aspectos que nos descubren estos resultados.

Quizás, el resultado más notorio que se pone en evidencia, sea que los egresados afrontan el inicio de estas enseñanzas con una percepción de sus propias competencias de niveles medios, siendo que, por su perfil académico anterior -una amplia mayoría provenientes de los Grados de Educación infantil y Educación Primaria- han recibido una escasa formación en estos ámbitos. Podría entenderse, por tanto, que el afrontamiento de tareas anteriores les ha llevado a desarrollar estas capacidades al menos a estos niveles. No obstante, también podría deberse al conocido como efecto Dunning-Kruger, un sesgo cognitivo por el que se tiende a sentir una ilusoria sensación de superioridad en algunas habilidades (Kruger y Dunning, 1999). Esta percepción, sin embargo, tendería a desaparecer a medida que se progresa y se adquiere una mayor cualificación, tomando conciencia real de lo que se sabe y lo que no, y llegando a manifestarse cierta inseguridad e incluso incapacidad para abordar una tarea y subestimar el nivel competencial; no es esto lo que sucede, ya que los estudiantes que han participado manifiestan de forma progresiva mayor competencia en cada una, aunque con matices, de las cinco seleccionadas, en la comparación longitudinal. 
Esta hipótesis explicativa nos llevaría hasta el segundo resultado reseñable en estos datos: no todas las competencias evolucionan igual. Los resultados parecen indicar que, algunas competencias, tienen un rápido desarrollo durante los primeros meses de formación, sufriendo luego cierto estancamiento. De esta forma, se encuentran que las diferencias significativas se producen únicamente entre el primer tiempo de medida y los tiempos 2 y 3 . Ocurre así con las competencias dirigidas a la revisión de textos científicos y de diseño de la investigación. Algunos autores (Miller y Geraci, 2011) han señalado que, en ocasiones, junto al desarrollo competencial se produce un desarrollo metacognitivo -el cual forma parte también de esta propuesta metodológica- que permite a los aprendices, una vez desarrolladas sus habilidades, alcanzar una meseta de logro que los lleve a incorporar la duda sobre su propia competencia. De esta forma, se explicaría el estancamiento una vez producido el primer salto cuantitativo en el desarrollo competencial, entre los tiempos dos y tres de algunas competencias.

Otras competencias, sin embargo, demuestran un desarrollo más tardío o lento, apareciendo las diferencias significativas únicamente en el último momento de medida. Ocurría así con las competencias referidas al análisis de datos y al manejo informático. Quizás esto esté indicando que determinadas habilidades requieren de un mayor tiempo de práctica para llegar a experimentar una mejora significativa, o incluso que requieren de una instrucción directa que modele los procesos cognitivos y procedimentales que requieren estas tareas (Raviolo, Ramírez y López, 2010), dado que los talleres competenciales más focalizados en ellas se llevaron a cabo durante el segundo cuatrimestre.

Finalmente, y derivado de todo lo anterior, el resultado que parece indiscutible a la luz de estos datos es que un trabajo coordinado, en equipos de docentes que, por otro lado trabajan en investigación también de forma conjunta, tiene éxito, en cuanto que tanto los talleres de cada asignatura como los dos talleres transversales, son percibidos por los estudiantes como actividades coherentes con la formación general que están recibiendo, lo que favorece el avance en la capacidad real del alumnado respecto a las distintas dimensiones de habilidades necesarias para el desarrollo de la labor investigadora. En este sentido, los resultados avalan que, como indican distintos autores (Aguirre, 1985; Biggs, 2011; Mayer, 2011) el trabajo coordinado repercute positivamente en la curva de aprendizaje, es decir, en la mejora de la eficacia con la que se da respuesta a una tarea a medida que esta se repite. La revisión de textos científicos y análisis crítico de argumentaciones, el manejo informático, el análisis de datos estadísticos, el diseño de la investigación, y la inferencia de conclusiones y elaboración de informes de investigación, resultan elementos clave en la formación de este alumnado que se percibe, cuando ingresa en el máster, con un nivel competencial insuficiente para abordar con éxito una tarea como el Trabajo Final de Máster que le será requerido después de los tres cuatrimestres que tiene su curso, y que debe expresar competencia general, aunque sea incipiente, para el trabajo investigador.. Solo el trabajo constante, en cada asignatura, y en los talleres transversales, mantiene la motivación y el estímulo necesario para que se produzcan los procesos metacognitivos que requiere la conciencia del progreso en autonomía y competencia para el avance en la planificación y desarrollo de la investigación. Distintos autores señalan, no obstante, que este proceso de aprendizaje requiere de un tiempo para acomodarse a la situación que rodea a la tarea, adaptarse a las demandas que les requiere y dar una respuesta ajustada; a medida que la tarea se repite, o que el aprendiz se hace más experto en ella, comienza a desarrollar un aprendizaje estratégico y a activar procesos 
metacognitivos lo que le permite ser más eficaz en la resolución de problemas tanto teóricos como prácticos. Desde esta mirada, el proyecto de innovación docente que aquí se ha ejecutado representa una potencialidad importante para el desarrollo de los estudios de postgrado en general, y para el Máster de Psicología Aplicada en particular, porque contribuye a la formación de su alumnado en las competencias cognitivas, procedimentales y metacognitivas necesarias para una verdadera formación investigadora.

\section{AGRADECIMIENTOS}

Agradecemos a todo el alumnado del Máster de Psicología Aplicada, por su continuidad y predisposición a la cumplimentación de los cuestionarios a lo largo de todo el proceso de investigación. Así mismo, nuestro agradecimiento a todos los profesores y profesoras participantes en este Proyecto de Innovación Docente, por su compromiso en el diseño y elaboración de los talleres implementados. Agradecemos también a la Universidad de Córdoba y al Plan de Innovación Docente por la financiación de este proyecto.

\section{REFERENCIAS}

Aguirre, A. (1985). Las curvas de aprendizaje y sus aplicaciones. Cuadernos de Ciencias Económicas y Empresariales, (16), 121-134.

Biggs, J. B. (2011). Teaching for quality learning at university: What the student does. U.K.: McGraw-Hill Education

European University Association (EUA) (2017). Annual Report 2016. Bruselas: EUA

Kruger, J., y Dunning, D. (1999). Unskilled and unaware of it: how difficulties in recognizing one's own incompetence lead to inflated self-assessments. Journal of personality and social psychology, 77(6), 1121.

Mayer, R. E. (2011). Applying the science of learning. Boston, MA: Pearson/Allyn \& Bacon.

Miller, T. M., y Geraci, L. (2011). Unskilled but aware: reinterpreting overconfidence in low-performing students. Journal of experimental psychology: learning, memory, and cognition, 37(2), 502.

Moral-López, A., Arrabal-Gómez, I., y González-López, I. (2010). Nuevas experiencias de evaluación estratégica en los centros educativos. La aplicación de una matriz DAFO en el centro de educación infantil y primaria "Mediterráneo" de Córdoba. Estudios sobre Educación, 18, 165-200

Observatorio del Sistema Universitario (2018). Informe Universidad 2000. Recuperado de http://www.observatoriuniversitari.org/es/files/2014/05/Bricall JM-2000Informe-Universidad-2000.pdf

Pérez-Gómez, A.I. (2010). Aprender a educar: nuevos desafíos para la formación de docentes. Revista interuniversitaria de formación del profesorado, (68), 37-60.

Raviolo, A., Ramírez, P., y López, E. A. (2010). Enseñanza y aprendizaje del concepto de modelo científico a través de analogías. Revista Eureka sobre Enseñanza y Divulgación de las Ciencias, 7(3).

Viejo, C. y Ortega-Ruiz (2018). Competencias para la Investigación: el Trabajo Fin de Máster y s potencialidad formativa. Revista de Innovación y Buenas Prácticas Docentes, 5, 46-56 DOI: https://doi.org/10.21071/ripadoc.v5i.10970 